\title{
$5,9-90$

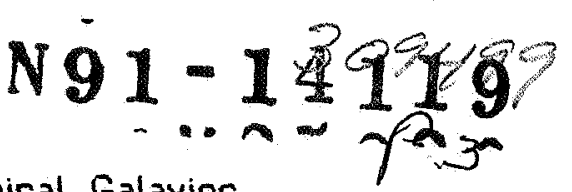

The Ratio of Molecular to Atomic Gas in Spiral Galaxies

as a function of Morphological Type

Patricia M. Knezek and Judith S. Young

University of Mass., Amherst, and Five College Radio Astronomy Observatory

\section{Introduction}

In order to gain an understanding of the global processes which influence cloud and star formation in disk galaxies, it is necessary to determine the relative amounts of atomic, molecular, and ionized gas both as a function of position in galaxies and from galaxy to galaxy. While atomic gas studies of galaxies have been underway for 30 years, the relative youth of the field of extragalactic molecular studies has meant that knowledge of the relative amounts of molecular and atomic gas in galaxies is limited by the relatively small number of galaxies observed in $\mathrm{CO}$.

Ever since the pioneering work in the 1950's and 1960's, it has been recognized that there is a morphological type dependence to the atomic gas content of galaxies. In particular, Roberts (1969) showed that the $\mathrm{HI}$ mass to blue luminosity ratio, $M(\mathrm{HI}) / \mathrm{L}_{\mathrm{B}}$, increases by a factor of 5 among spiral galaxies from types Sa through Scd. With observations of the $C D$ distributions in over 200 galaxies now completed as part of the FCRRD Extragalactic CO Survey (Young et al. 1989), we are finally in a position to determine the type dependence of the molecular content of spiral galaxies, along with the ratio of molecular to atomic gas as a function of type. Do late type spirals really have more gas than early types when the molecular gas content is included?

\section{Sample and Rnalysis}

The galaxies observed as part of the FCRAO Extragalactic CO Survey are either (1) brighter than $B_{T}^{\circ}=12.5$ in the blue, or (2) brighter than $20 \mathrm{Jy}$ at $100 \mu \mathrm{m}$. From major axis $\mathrm{CD}$ observations at 45" resolution and spacing in over 200 galaxies, we have derived global $c 0$ fluxes (cf. Kenney and Young 1988); $\mathrm{H}_{2}$ masses were derived using the conversion factor $N\left(H_{2}\right) / I C O=2.8 \times 10^{20} \mathrm{~cm}^{-2} /\left(\mathrm{K} \mathrm{km} \mathrm{s}^{-1}\right.$ ) (Bloemen et al. 1986). HI masses for the sample galaxies were taken from Huchtmeier et al. (1983), and morphological types are from RC2.

\section{Results and Discussion}

Within our galaxy sample, we confirm the previously found trend of $M(H I) / L_{B}$ to increase with increasing type from Sab-Sdm. We find the mean ratio of $M\left(\mathrm{H}_{2}\right) / L_{B}$ to be roughly constant 
$-$

for types Sa-Sc, with a decrease of a factor of $>3$ for types Scd-Sdm. The combination of these two effects is that the mean value of the ratio of molecular to atomic gas decreases smoothly by a factor of $\sim 20$ as a function of morphological type for types Sa-So as shoun in Figure 1.

We have verified that the observed $\mathrm{H}_{2} / \mathrm{HI}$ ratio variation with type is not due to the inclusion of Virgo cluster spiral galaxies, many of which are $\mathrm{HI}$-deficient early type galaxies with high $\mathrm{H}_{2} / \mathrm{HI}$ ratios (van Gorkom and Kotanyi 1985; Kenney and Young 1986, 1988; Stark et al. 1986). We have also verified that the observed decrease of $\mathrm{H}_{2} / \mathrm{HI}$ is not the result of a Malmquist bias, since the observed trend is also found in the subset of galaxies more nearby than the Virgo cluster. We argue that metallicity variations with type among the galaxies in our sample will be small, since the mean mass for types Sa-Scd changes by only a factor of 4 and the $[\mathrm{O} / \mathrm{H}]$ ratio shows almost no variation with mass for spirals between $10^{10}$ and $10^{12} M_{\odot}$ (Pagel and Edmunds 1981).

In order to ascertain whether the observed $\mathrm{H}_{2} / \mathrm{HI}$ variation is due to systematic changes in molecular cloud properties, and thereby in the $\mathrm{CO} \rightarrow \mathrm{H}_{2}$ conversion as a function of morphological type, we have determined the mean dust temperature for the galaxies in our sample as a function of type. We find $T_{\text {dust }}$ to be 1.2 times higher in the Sa-Sab galaxies in the sample than in the $5 b-5 d m$ galaxies. If we assume that the gas and dust temperatures are equal, then we will have overestimated $M\left(\mathrm{H}_{2}\right)$ in the early type spirals by a factor of 1.2 , since $M\left(H_{2}\right)=L_{C O} \sqrt{p} / T_{\text {gas }}$ (Dickman, Snell, and Schloerb 1986; Maloney and Black 1988; Elmegreen 1989). Correcting the observed $M\left(\mathrm{H}_{2}\right) / M(\mathrm{HI})$ ratio in each galaxy by the observed dust temperature leaves a factor of 17 variation in the ratio $M\left(\mathrm{H}_{2}\right) / M(\mathrm{HI})$ with morphological type.

\section{Conclusions}

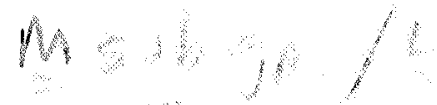

We conclude that there is more than an order of magnitude decrease in the ratio of molecular to atomic gas mass as a function of morphological type from Sa-Sd; an average Sa galaxy has more molecular than atomic gas, and an average Sc has less. Therefore, the total interstellar gas mass to blue luminosity ratio, $\mathrm{Mgas}_{B}$ increases by less than a factor of two as a function of type from Sa-Sd. The dominant effect we find is that the phase of the gas in the cool ISM varies along the Hubble sequence.

We suggest that the more massive and centrally concentrated galaxies are able to achieve a molecular-dominated ISM through the collection of more gas in the potential. That gas may then form molecular clouds when a critical density is exceeded. The picture which these observations support is one in which the conversion of atomic gas to molecular gas is a global process which depends on large scale dynamics (cf. Uyse 1986).

Among interacting and merging systems, we find considerable scatter in the $M\left(\mathrm{H}_{2}\right) / \mathrm{M}(\mathrm{HI})$ ratio, with the mean ratio similar to that in the early type galaxies These high global ratio of molecular to atomic gas could result from the removal of $\mathrm{HI}$ gas, the enhanced conversion of $\mathrm{HI}$ into $\mathrm{H}_{2}$, or both. 


\section{References}

Bloemen, J.B.G., et al. 1986, Astr.Ap., 154, 25.

Dickman, R.L., Snell, R.L., and Schloerb, F.P. 1986, Ap.J., 309, 326.

Elmegreen, B.G. 1989, Ap.J., 338, 178.

Huchtmeier, W.K., Richter, 0.-G., Bohnenstengel, H-D., and Hauschildt, M. 1983, ESO preprint.

Kenney, J.D.P., and Young, J.S. 1988, Bp.J.Suppl., 66, 261.

Maloney, P., and Black, J.H. 1988, Ap.J., 325, 389.

Pagel, B.E.J., and Edmunds, M.G. 1981, Ann.Rev.Astr.Ap., 19, 77.

Roberts, M.S. 1969, A.J., 74, 859.

van Gorkom, J., and Kotanyi, K. 1985, in ESO Workshop on the Virgo Cluster of Galaxies, p. 61. Wyse, R. 1986, Ap.J.(Letters), 311, L41.

Young, J.S., Claussen, M., Devereux, N., Huang, Y., Kenney, J., Knezek, P., Tacconi, L., TacconiGarman, L., Schloerb, P., Viscuso, P., and Xie, 5. 1989, in preparation.

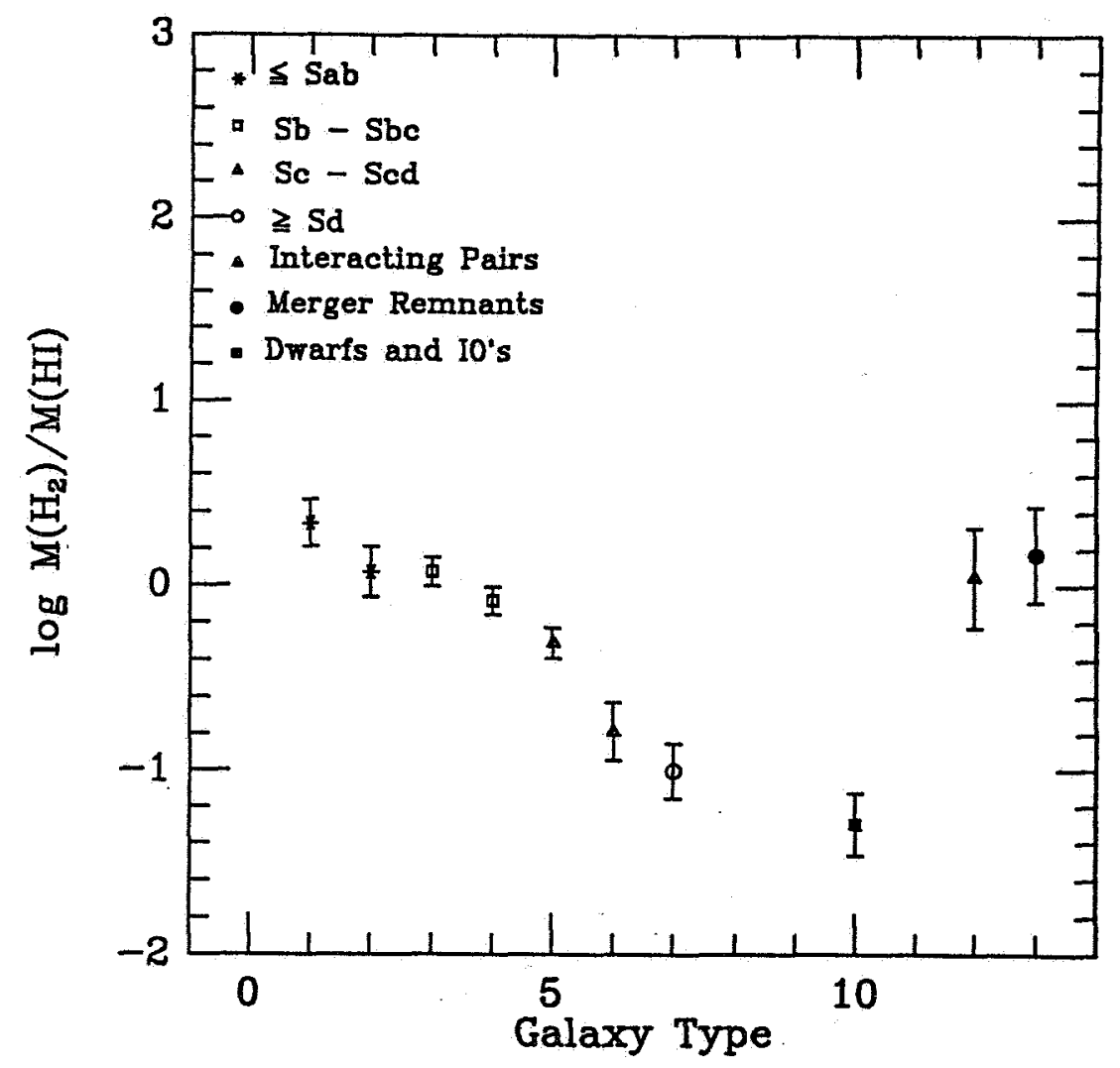

Figure 1. Ratio of molecular to atomic gas mass as a function of morphological type among 200 galaxies. We have combined RC2 types 0 and 1, types 7 and 8 , and types 9 and 10 . Also included in category 9-10 are duarf galaxies and 10 galaxies (i.e. M82). Type 12 represents interacting galaxy pairs and type 13 represents merger remnants. The error bars shown represent the error in the mean $M\left(\mathrm{H}_{2}\right) / M(\mathrm{HI})$ ratio for each type. 